\title{
The Chalker-Coddington Network Model is Quantum Critical
}

\author{
J. B. Marston and Shan-Wen Tsai \\ Department of Physics, Brown University, Providence, Rhode Island 02912-1843
}

(July 5, 2018)

\begin{abstract}
We show that the localization transition in the integer quantum Hall effect as described by the Chalker-Coddington network model is quantum critical. We first map the anisotropic network model to the problem of diagonalizing a one-dimensional non-Hermitian non-compact supersymmetric lattice Hamiltonian of interacting bosons and fermions. Its behavior is investigated numerically using the density matrix renormalization group method, and critical behavior is found at the plateau transition. This result is confirmed by a generalization of the Lieb-Schultz-Mattis theorem.
\end{abstract}

PACS numbers: 73.40.Hm, 71.30.+h, 72.16.Rn, 75.10.Jm

Transitions between plateaus in the integer quantum Hall effect provide the clearest example of quantumcritical behavior in a disordered system. Understanding such critical points is a challenging problem because fluctuations occur over many decades of length and time scales, and averages over different realizations of the disorder must be carried out. Critical behavior was predicted by Levine, Libby, and Pruisken [1] and was observed experimentally by Wei et al. [2] for temperatures close to the critical point at absolute zero. Progress toward a theoretical understanding of the plateau transition was achieved with the introduction of a quantum tunneling network model by Chalker and Coddington [3]. Subsequent numerical studies 䐗 of the ChalkerCoddington model yielded values for the correlation length exponent $\nu \approx 2.3$ which were consistent with experiments. To the best of our knowledge, however, there has been no exact proof that the Chalker-Coddington model is quantum critical.

The method of supersymmetry (SUSY) can be used to analytically carry out disorder averages [5 9]. We apply the method to the anisotropic network model and then use the Density Matrix Renormalization Group (DMRG) algorithm [10] to study the resulting spin chain. Unlike usual spin chains such as the spin-1/2 Heisenberg antiferromagnet, the on-site Hilbert space in the SUSY chain is infinite-dimensional. To access the critical point numerically therefore requires a double-extrapolation to large on-site Hilbert spaces and to large chain lengths. We use our numerical results to motivate a generalization of the theorem of Lieb, Schultz and Mattis (LSM) which confirms quantum criticality.

1. SUSY Spin-Chain for the Network Model. The anisotropic Chalker-Coddington model can be represented 11, 12 by an independent-particle Hamiltonian which describes a chain of $L$ (even) edge states alternating in propagation forward and backward in imaginary time $\tau$. Random complex tunneling amplitudes $t_{j}(\tau)$ between adjacent edge states account for the AharonovBohm phases accumulated by the electrons as they circulate around equipotential contours of the random po- tential:

$$
\begin{array}{r}
H_{i p}=\int d \tau\left\{\sum_{j=0}^{L-1}(-1)^{j} \psi_{j}^{\dagger}(\tau) \mathrm{i} \partial_{\tau} \psi_{j}(\tau)\right. \\
\left.-\sum_{j=0}^{L-2}\left[t_{j}(\tau) \psi_{j}^{\dagger}(\tau) \psi_{j+1}(\tau)+t_{j}^{*}(\tau) \psi_{j+1}^{\dagger}(\tau) \psi_{j}(\tau)\right]\right\} .
\end{array}
$$

The $(-1)^{j}$ term has its origin in the alternating propagation of adjacent edge states and this factor reappears several times in the equations which follow. The disorder average of the tunneling amplitudes is given by

$$
\begin{aligned}
\overline{t_{j}^{*}(\tau) t_{j^{\prime}}\left(\tau^{\prime}\right)} & =J_{j} \delta_{j, j^{\prime}} \delta\left(\tau-\tau^{\prime}\right), \\
J_{j} & =\left[1+(-1)^{j} R\right] .
\end{aligned}
$$

The relevant dimerization parameter $R= \pm 1$ deep inside the plateaus; the transition between the plateaus occurs at $R=0$. Disorder averaging of the corresponding functional integral is made possible with the use of SUSY 113, 14 as the partition function $Z=1$ for each realization of the disorder. Transfer matrix formalism can be used to resolve normal-ordering ambiguities [15, 16 and the resulting effective SUSY Hamiltonian may then be extracted. It describes interacting spin-up and spindown fermions $c_{\sigma}$ and bosons $b_{\sigma}$ (two spin species are introduced to permit the calculation of the disorderaveraged product of retarded and advanced Green's functions which determines the conductivity):

$$
\begin{aligned}
H= & \sum_{j=0}^{L-2} J_{j}\left[\sum_{a=1}^{8} g_{a} S_{j}^{a} S_{j+1}^{a}+(-1)^{j} \sum_{a=9}^{16} g_{a} S_{j}^{a} S_{j+1}^{a}\right] \\
& +\eta \sum_{j=0}^{L-1}\left[S_{j}^{1}+S_{j}^{2}+S_{j}^{5}+S_{j}^{6}\right] .
\end{aligned}
$$

Parameter $\eta>0$ ensures convergence of the non-compact bosonic sector and defines the advanced and retarded propagators; also the signs $g_{a}$ are given by:

$$
g_{a}=\left\{\begin{array}{l}
1 ; a=1,2,10,12,14,16 \\
-1 ; a=3, \ldots, 9,11,13,15 .
\end{array}\right.
$$


In Eq. 3 we have introduced 16 spin operators, the components of a $4 \times 4$ superspin matrix:

$$
\begin{aligned}
S^{1} \equiv b_{\uparrow}^{\dagger} b_{\uparrow}+1 / 2 & S^{9} \equiv c_{\downarrow}^{\dagger} b_{\downarrow} \\
S^{2} \equiv b_{\downarrow}^{\dagger} b_{\downarrow}+1 / 2 & S^{10} \equiv c_{\uparrow}^{\dagger} b_{\uparrow} \\
S^{3} \equiv b_{\uparrow}^{\dagger} b_{\downarrow}^{\dagger} & S^{11} \equiv b_{\downarrow}^{\dagger} c_{\downarrow} \\
S^{4} \equiv b_{\downarrow} b_{\uparrow} & S^{12} \equiv b_{\uparrow}^{\dagger} c_{\uparrow} \\
S^{5} \equiv c_{\uparrow}^{\dagger} c_{\uparrow}-1 / 2 & S^{13} \equiv b_{\downarrow} c_{\uparrow} \\
S^{6} \equiv c_{\downarrow}^{\dagger} c_{\downarrow}-1 / 2 & S^{14} \equiv b_{\uparrow} c_{\downarrow} \\
S^{7} \equiv c_{\uparrow}^{\dagger} c_{\downarrow}^{\dagger} & S^{15} \equiv b_{\downarrow}^{\dagger} c_{\uparrow}^{\dagger} \\
S^{8} \equiv c_{\downarrow} c_{\uparrow} & S^{16} \equiv b_{\uparrow}^{\dagger} c_{\downarrow}^{\dagger} .
\end{aligned}
$$

Bosonic-valued operators $S^{1}, \ldots, S^{8}$ make up the symmetric sector of the Hamiltonian while fermion-valued operators $S^{9}, \ldots, S^{16}$ are in the antisymmetric sector. Despite the fact that $H$ is non-Hermitian, it only has real-valued eigenvalues.

The Hamiltonian commutes with four (fermion-valued) supersymmetry generators, $\left[H, Q_{1 \sigma}\right]=\left[H, Q_{2 \sigma}\right]=0$, where

$$
\begin{aligned}
Q_{1 \sigma} & \equiv \sum_{j}\left[b_{j \sigma}^{\dagger} c_{j \sigma}-(-1)^{j} c_{j \sigma}^{\dagger} b_{j \sigma}\right] . \\
Q_{2 \sigma} & \equiv \sum_{j}\left[(-1)^{j} b_{j \sigma}^{\dagger} c_{j \sigma}+c_{j \sigma}^{\dagger} b_{j \sigma}\right] .
\end{aligned}
$$

It is not difficult to see that the supersymmetric Hamiltonian must have a unique, zero-energy, ground state. The right and left (ground) eigenstates are therefore annihilated by the Hamiltonian: $H\left|\Psi_{0}\right\rangle=\left\langle\Psi_{0}\right| H=0$. Also, the ground state is annihilated by the SUSY charges: $Q_{1 \sigma}\left|\Psi_{0}\right\rangle=Q_{2 \sigma}\left|\Psi_{0}\right\rangle=0$. All excited states appear in quartets or larger multiples of 4 , half with odd total fermion content, and these cancel out in the partition function by virtue of the supertrace:

$$
Z=\mathrm{S} \operatorname{Tr} e^{-\beta H} \equiv \operatorname{Tr}(-1)^{N_{c}} e^{-\beta H}=1,
$$

where $N_{c}$ is the total number of fermions. In the limit of $\eta \rightarrow \infty,\left|\Psi_{0}\right\rangle \rightarrow|0\rangle$. For finite $\eta>0$, however, it is a remarkable fact that the normalized ground state is a superposition of the vacuum state with unit amplitude and an infinite number of zero-norm many-body states $|J\rangle$ with differing total number of particles [17]:

$$
\left|\Psi_{0}\right\rangle=|0\rangle+\sum_{J=1}^{\infty} a_{J}(\eta)|J\rangle ; \quad\langle I \mid J\rangle=0 \forall I, J>0 .
$$

This result can be verified directly by observing that $\left|\Psi_{0}\right\rangle=\lim _{\beta \rightarrow \infty} e^{-\beta H}|0\rangle$ and application of powers of $H$ to the vacuum state yields only zero-norm states. We have also checked numerically, for finite length chains, that the vacuum state has unit amplitude when the Hilbert space is truncated in a way which respect supersymmetry (see below). It is useful to contrast the complicated ground state of the non-Hermitian SUSY Hamiltonian with the ground state of the Hermitian SUSY ferromagnet which describes a chiral metal with all edge states propagating in the same direction [9]. Backscattering is absent, localization cannot occur, and the ground state of the SUSY ferromagnet is simply the vacuum state.

2. DMRG Analysis. To simply show that the density of states (DOS) is non-vanishing it suffices to remove one of the spin sectors, for example the $\downarrow$-spins. The remaining $\uparrow$-spin degrees of freedom are then compact, the ground state is the vacuum state $|0\rangle$, the DOS is proportional to $\left\langle S^{1}\right\rangle=-\left\langle S^{5}\right\rangle=1 / 2$, and the Hamiltonian can be exactly diagonalized [14]. When both spin sectors are included the theory is non-compact and highly nontrivial. To make further progress we employ the infinitesize DMRG method [10]. The Hilbert space is first constructed systematically on each site by repeated action of the double-creation operator $S^{3} \equiv b_{\uparrow}^{\dagger} b_{\downarrow}^{\dagger}$. Introducing the integer level index $n=0,1,2, \ldots$ we add to the vacuum state $|0\rangle$ a tower of states built out of the quartets:

$$
\begin{aligned}
& |4 n+1\rangle \equiv \frac{1}{n !}\left(b_{\uparrow}^{\dagger} b_{\downarrow}^{\dagger}\right)^{n} c_{\uparrow}^{\dagger} c_{\downarrow}^{\dagger}|0\rangle \\
& |4 n+2\rangle \equiv \frac{1}{\sqrt{n !(n+1) !}}\left(b_{\uparrow}^{\dagger} b_{\downarrow}^{\dagger}\right)^{n} b_{\uparrow}^{\dagger} c_{\downarrow}^{\dagger}|0\rangle \\
& |4 n+3\rangle \equiv \frac{1}{\sqrt{n !(n+1) !}}\left(b_{\uparrow}^{\dagger} b_{\downarrow}^{\dagger}\right)^{n} c_{\uparrow}^{\dagger} b_{\downarrow}^{\dagger}|0\rangle \\
& |4 n+4\rangle \equiv \frac{1}{(n+1) !}\left(b_{\uparrow}^{\dagger} b_{\downarrow}^{\dagger}\right)^{n} b_{\uparrow}^{\dagger} b_{\downarrow}^{\dagger}|0\rangle .
\end{aligned}
$$

Truncations with $D=4 n+1$ states preserve supersymmetry, as the SUSY generators, Eq. 6, intermix the quartet of states, Eq. 9, separately within each level of the tower without changing the total number of particles. The DOS remains unchanged, and the ground state energy is exactly zero, providing a valuable check on the accuracy of the DMRG algorithm which incurs errors when, as the chain length increases, the Hilbert spaces of the blocks grow beyond the finite limit of $M$ states. Increasing $M$ up to limits set by machine memory and speed yields systematic improvement in the accuracy of the DMRG algorithm. In results reported below we have checked that $M$ is sufficiently large to ensure adequate accuracy; even in the challenging case of $D=13$ and $M=170$ the ground state, when targeted, had an energy which deviated from zero by less than 0.003 .

Reduced density matrices for the two augmented blocks, each of Hilbert space size $D \times M$, are formed by computing a partial trace over half the chain. For the 
left half of the chain the density matrix is given by:

$$
\rho_{i j}=\sum_{i^{\prime}=1}^{D M} \Psi_{i i^{\prime}} \Psi_{j i^{\prime}}
$$

Here $\Psi_{i i^{\prime}} \equiv\left\langle i, i^{\prime} \mid \Psi\right\rangle$ are the real-valued matrix elements of the targeted many-body wavefunction projected onto a basis of states labeled by unprimed Roman index $i$ which covers the left half of the chain and primed index $i^{\prime}$ which covers the right half. To compute the gap, $\Psi$ is chosen to be one of the lowest-lying right eigenstates of $H$. All of the eigenvalues of $\rho$ are real and positive; these are interpreted as probabilities and the $(D-1) M$ least probable states are thrown away. The desired $D \rightarrow \infty$ limit can be reached by extrapolation, Fig. 1. Fig. 2 1 shows that the gap is non-zero in the thermodynamic limit for finite fixed $D=4 n+1$ (solid lines), but as shown in Fig. 11 it approaches zero in the $D \rightarrow \infty$ limit as expected.

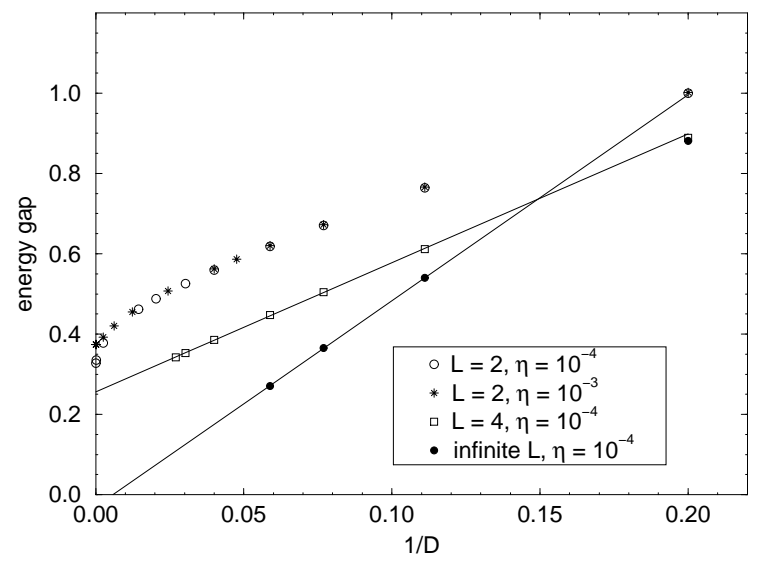

FIG. 1. Gaps to the lowest excited state for chains with open boundary conditions and $R=0$. The gap for the two-site problem was obtained by diagonalizing Eq. 61 of Ref. 16. A straight line is fit to the $L=4$ points (with $M=D)$. Also shown are gaps at $L \rightarrow \infty$ which are obtained from the extrapolations presented in Fig. 2 for the supersymmetric truncations $D=5,9,13$, and 17 . A straight line is fit to the last three points.

Also of interest are non-supersymmetric truncations $D=4 n+2$, with the state $|4 n+1\rangle$ selected as the final state at the top of the tower. The special case of no bosons, $D=2$, with on-site states $\left\{|0\rangle, c_{\uparrow}^{\dagger} c_{\downarrow}^{\dagger}|0\rangle\right\}$ corresponds to the ordinary spin- $1 / 2$ Heisenberg antiferromagnet as can be verified by making separate particlehole transformations on the even and odd sublattices: $c_{2 j \uparrow} \leftrightarrow c_{2 j \uparrow}^{\dagger}$ and $c_{2 j+1 \downarrow} \leftrightarrow c_{2 j+1 \downarrow}^{\dagger}$; consequently the gap vanishes in this case. As seen in Fig. 2 the gap also vanishes for $D=6$. We prove below that all truncations with $D=4 n+2$ are gapless.

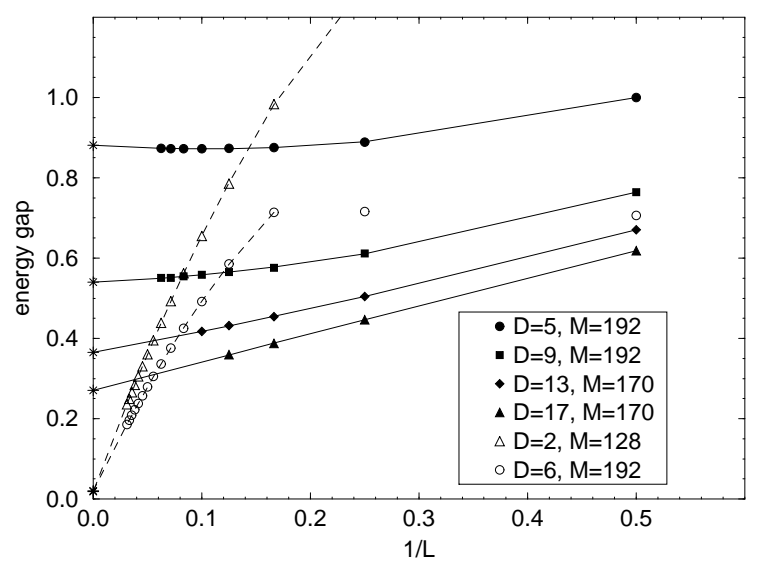

FIG. 2. Gaps, for $\eta=10^{-4}$, extrapolated to $L \rightarrow \infty$. Truncations which respect supersymmetry (solid lines) and non-supersymmetric truncations (dashed lines) are plotted. Points at $L=\infty$ are obtained by fitting the gap to the form $a+b / L+c / L^{2}$.

3. Lieb-Schultz-Mattis Theorem. For half-odd-integer spin antiferromagnets on a periodic chain of length $L$ sites $\left(\vec{S}_{L} \equiv \vec{S}_{0}\right)$ LSM showed [18 that either (1) the ground state is degenerate or (2) there are gapless spin excitations in the thermodynamic limit $L \rightarrow \infty$. LSM employed a variational argument by introducing the unitary slow-twist operator,

$$
U \equiv \exp \left\{\frac{2 \pi i}{L} \sum_{j=0}^{L} j S_{j}^{z}\right\}
$$

which has the property that $U^{\dagger}[H, U]=O(1 / L)$. Now $\left\langle\Psi_{0}|U| \Psi_{0}\right\rangle=0$ because $U \rightarrow-U$ under parity of reflection about the middle site $(j \leftrightarrow L-j)$ combined with a rotation of 180 degrees about the y-axis $\left(S^{z} \leftrightarrow-S^{z}\right)$. So $U$ either creates a low-energy excitation above a unique ground state or mixes degenerate ground states. In contrast, for integer spins $U \rightarrow U$ under parity and $U$ does not, in general, create a low-energy excitation or switch degenerate ground states.

In the SUSY problem we are able to make a stronger statement, because we know that the ground state is unique by supersymmetry. The natural generalization of the LSM slow-twist operator for the SUSY chain is:

$$
U \equiv \exp \left\{\frac{\pi i}{L} \sum_{j=0}^{L} j(-1)^{j}\left[n_{c}(j)+n_{b}(j)-1\right]\right\},
$$

where $n_{b}(j) \equiv b_{j \sigma}^{\dagger} b_{j \sigma}$ is the number of bosons on site $j$ and $n_{c}(j) \equiv c_{j \sigma}^{\dagger} c_{j \sigma}$ is the number of fermions. It reduces, in the $D=2$ limit of no bosons, and after the particlehole transformation is taken, to the usual LSM operator Eq. 11. In the ground state, the sum $n_{c}(j)+n_{b}(j)$ 
is always an even number, so $U$ respects the periodic boundary condition and it also has the desired property $U^{\dagger}[H, U]=O(1 / L)$. The canonical parity transformation now takes the form:

$$
\begin{aligned}
j & \leftrightarrow L-j \\
(-1)^{j} & \leftrightarrow(-1)^{j} \\
c_{j \sigma}^{\dagger} & \leftrightarrow c_{L-j \sigma} \\
b_{j \uparrow} & \leftrightarrow b_{L-j \downarrow}
\end{aligned}
$$

again reducing in the absence of bosons to the usual LSM parity operation. The supersymmetric Hamiltonian is invariant under this operation, but only at the presumed critical point $R=\eta=0$. The slow-twist operator $U$, however, changes form because while $n_{c} \rightarrow 2-n_{c}$, the number of bosons $n_{b}$ remains invariant:

$$
U \rightarrow-\exp \left\{\frac{\pi i}{L} \sum_{j=0}^{L} j(-1)^{j}\left[n_{c}(j)-n_{b}(j)-1\right]\right\} .
$$

However, $U$ is invariant under global supersymmetry rotations, $\left[Q_{1 \sigma}, U\right]=\left[Q_{2 \sigma}, U\right]=0$. As only the ground state is a SUSY singlet, $U$ cannot create a low-energy excitation for truncations which respect SUSY, consistent with the above DMRG results. Indeed, from Eq. 8 it follows that $\left|\left\langle\Psi_{0}|U| \Psi_{0}\right\rangle\right|=1$ and thus $U\left|\Psi_{0}\right\rangle$ does not contain a component orthogonal to the ground state $\left|\Psi_{0}\right\rangle$. (This can be viewed as an alternative proof of Eq. 8.) For non-SUSY truncations $D=4 n+2$, however, the ground state does not obey Eq. 8; instead $\left|\left\langle\Psi_{0}|U| \Psi_{0}\right\rangle\right|<1$ as can be verified either directly for small chains, and in the special case $D=2$ of no bosons (for which $\left\langle\Psi_{0}|U| \Psi_{0}\right\rangle=0$ ), or by appealing to the fact that $U$ changes form under the parity operation, Eq. 14. For sufficiently large $D$ and $\eta>0$ the ground state approaches the SUSY ground state and is thus unique; therefore $U$ creates low-energy excitations [19], and chains with the non-SUSY truncation $D=4 n+2$ are gapless in the thermodynamic limit.

Before examining the physically relevant $D \rightarrow \infty$ limit, first consider the large spin limit of one-dimensional nearest-neighbor Heisenberg antiferromagnets. Chains with spin $S=n+1 / 2$ (and even-numbered $D=2 S+1=$ $2 n+2)$ are gapless for all integer $n \geq 0$. Chains with $S=n+1$ (and odd-numbered $D=2 n+3$ ) are Haldane gapped, but this gap must vanish in the limit of large spin to accord with the gaplessness of the half-odd-integer in the $n \rightarrow \infty$ limit. This reasoning can be checked by a simple renormalization-group argument using the beta function for the non-linear $\sigma$-model which shows that the gap for integer-spin chains vanishes as $e^{-\pi S}$. Likewise, for $\eta=0^{+}$, continuity requires that SUSY truncations with odd-numbered $D=4 n+1$ must converge to the gapless behavior exhibited by the non-SUSY even-numbered $D=4 n+2$ truncations in the $n \rightarrow \infty$ limit. Hence the Chalker-Coddington model is quantum critical at $R=0$.
We thank W. Craig, S. Das Sarma, M.P.A. Fisher, I. Gruzberg, G. Guralnik, V. Gurarie, B. Huckestein, J. Kondev, A. W. W. Ludwig, T. Senthil, and Z. Wang for helpful comments. J.B.M. thanks the Institute for Theoretical Physics for its hospitality during the completion of this work. This research was supported in part by the NSF under Grants Nos. DMR-9357613, DMR-9712391 and PHY94-07194. Computations were carried out in $\mathrm{C}++$ on Cray PVP machines at the Theoretical Physics Computing Facility at Brown University.

[1] H. Levine, S. B. Libby, and A. M. M. Pruisken, Phys. Rev. Lett. 51, 1915 (1983); A. M. M. Pruisken in The Quantum Hall Effect edited by R. E. Prange and S. M. Girvin (Springer-Verlag, New York, 1990).

[2] H. P. Wei, D. C. Tsui, M. A. Paalanen, and A. M. M. Pruisken, Phys. Rev. Lett. 61, 1294 (1988). See, however, D. Shahar et al., Solid State Comm. 107, 19 (1998).

[3] J. T. Chalker and P. D. Coddington, J. Phys. C 21, 2665 (1988).

[4] D.-H. Lee, Z. Wang, and S. A. Kivelson, Phys. Rev. Lett. 70, 4130 (1993); D. K. K. Lee and J. T. Chalker, Phys. Rev. Lett. 72, 1510 (1994).

[5] A. McKane, Phys. Lett. 76A, 22 (1980).

[6] K. B. Efetov, Adv. Phys. 32, 53 (1983).

[7] I. Affleck, J. Phys. C 17, 2323 (1984).

[8] M. R. Zirnbauer, Annalen der Physik 3, 513 (1994).

[9] L. Balents, M. P. A. Fisher, and M. Zirnbauer, Nucl. Phys. B483, 601 (1997).

[10] S. R. White, Phys. Rev. Lett. 69, 2863 (1992); Phys. Rev. B 48, 10,345 (1993).

[11] Dung-Hai Lee, Phys. Rev. B 50, 10,788 (1994).

[12] Dung-Hai Lee and Ziqiang Wang, Phil. Mag. Lett. 73, 145 (1996).

[13] M. R. Zirnbauer, J. Math. Phys. 38, 2007 (1997).

[14] J. Kondev and J. B. Marston, Nucl. Phys. B 497, 639 (1997). We have corrected a mistake in this paper due to the normal-ordering ambiguity, and thank L. Balents for pointing out the error to us.

[15] I. A. Gruzberg, N. Read, and S. Sachdev, Phys. Rev. B 55, 10,593 (1997).

[16] H. Mathur, Phys. Rev. B 56, 15,794 (1997).

[17] A similar result holds for a one-dimensional model of localization. See: L. Balents and M. P. A. Fisher, Phys. Rev. B 56, 12970 (1997).

[18] E. Lieb, T. D. Schultz, and D. C. Mattis, Ann. Phys. 16, 407 (1961).

[19] The state $U\left|\Psi_{0}\right\rangle$ is a superposition of eigenstates, each of non-negative norm, because the non-SUSY truncated Hamiltonian may be viewed as the SUSY truncation plus a Hermitian perturbation. The zero-norm SUSY states acquire non-negative norm and an upper bound on the energy of the excitation can be established. 